\title{
Representing auditory space
}

\author{
SANDRA R. PALEF and RAND B. NICKERSON \\ Ontario Institute for Studies in Education, University of Toronto, Toronto, Canada M5S IV6
}

\begin{abstract}
Auditory space has been characterized as an entity without bound or dimension, as opposed to visual space which is limited in three dimensions. While there is evidence that visual space may be represented mentally in terms of contrastive values on these dimensions, no evidence exists concerning the representation of auditory space. Two experiments used an auditory Stroop-like task to investigate (1) whether the linguistic code for auditory space is comprised of component dimensions as in vision or whether it is unitary, and (2) whether auditory space can also be encoded in a nonlinguistic fashion. Subjects were required to respond to the spatial location of a locative term whose meaning could be congruent, incongruent, or neutral with respect to its location. The findings pointed to the conclusion that when subjects are encouraged to code auditory space linguistically, the code is an undifferentiated symbol or name. Furthermore, some tentative evidence existed that auditory space may also be encoded in a nonlinguistic manner.
\end{abstract}

\begin{abstract}
A dark illimitable ocean, without bound, without dimension, where length, breadth and height and Time and place are lost.
\end{abstract}

Milton's classical description of auditory space as an entity without fixed boundaries contrasts sharply with our notion of visual space as limited in three dimensions. We appear to code visual space in terms of axes which are appropriate to the purposes or functions of the perceiver. Our vertical bias (above/ below) is determined by gravity and a reference plane at ground level; the primary horizontal (front/back) is thought to be related to the orientation of our perceptual apparatus; and the secondary horizontal (left/right) is related to our bilateral nature. Notice that we code these dimensions in terms of contrastive pairs. Visual space appears to be perceived and represented in terms of a discrete, notational symbol system. On the other hand, an essential feature of sound is that its locality is normally irrelevant. Rather, it seems to fill auditory space (Williams, 1955). The perception of auditory space has been characterized as continuous, simultaneous, allinclusive (McLuhan, 1964), rather than involving the breakup of experience into lineal sequences of discrete units as in vision.

This popular distinction between the perception of visual and auditory space is an intriguing one, but one which has received little empirical investigation.

This work was supported by Grant S73-0402 from Canada Council to the first author. Address correspondence to Sandra $R$. Palef, Department of Applied Psychology, Ontario Institute for Studies in Education, University of Toronto, 252 Bloor Street West, Toronto, Canada M5S 1 V6.
The representation of visual space has been studied by means of an investigation of the language of visual space (Clark, 1973; Clark, Carpenter \& Just, 1973; Leech, 1969; Olson \& Bialystock, in press). Clark (1973) rejected the Whorfian notion that the linguistic system for space determines how visual space is perceived and coded in favor of the assumption that both linguistic and perceptual representations arise independently from a basic conceptual organization of space. On the other hand, Olson and Bialystock (in press) argue that the two are dependent systems, with the linguistic derived from the perceptual. In any case, the language of space is believed to be an indication of how visual space is represented. For example, since the language of space is composed of contrastive pairs of terms like front/back, one of which is unmarked or positive while the other is marked or negative, the argument is that visual space is initially perceived and represented in this manner. Indeed, Olson and Bialystock (in press) have argued that a complete account of the linguistic features comprising the language of space will provide a description of the representational structures for visual space.

Clark et al. (1973) provided evidence that linguistic properties reflect visual perceptual codes. For example, they point out that, according to Greenberg's (1966) criteria of linguistic complexity, the set of terms deep-shallow is linguistically more complex than tall-short. To demonstrate that this property is true of perceptual codes as well, they showed subjects a pair of rectangles, and required them to answer questions such as "which is taller (shorter, deeper, shallower)?" In one condition, picture encoding time was measured independently of linguistic encoding time by presenting the rectangles 
$1.5 \mathrm{sec}$ after the question and timing the subject's response from the onset of the picture. It was found that comparative judgments about the vertical dimension of the rectangles took approximately $30 \mathrm{msec}$ longer if the question referred to depth than if it referred to height. The effect was attributable to the perceptual coding and comparison processes rather than linguistic encoding of the question. Clark suggested that encoding a picture in terms of depth requires construction of representational components not required by height, and this requires additional time. Further evidence for the notion that linguistically more complex features take longer to encode from pictures comes from the repeated finding that marked adjectives are encoded more slowly than their unmarked counterparts, presumably because the former are more complex.

Another kind of evidence favoring the view that pictorial and linguistic representations of space share common components is the finding of Stroop-like interference effects between spatial locations and locative terms (see Stroop, 1935). In one such study, Seymour (1973) showed subjects one of the words above, below, left, and right, which was located ABOVE, BELOW, LEFT, or RIGHT with respect to a dot. When subjects named the location of the word relative to the dot, reaction time was faster when the word meaning and location were congruent than when they were incongruent. Moreover, it was argued that if spatial locations are semantically represented in the same manner as spatial words, then incongruent reaction times will show maximal delays when the components of the incongruent word code overlap with the components of the picture code. Assuming that above and below share a vertical feature while left and right share the horizontal, the word above should provide more interference on a decision that it is located BELOW a dot than should the words left or right, and the word left should interfere more with the location RIGHT than should the words above or below. This prediction was confirmed, although there were asymmetries in the effects, i.e., an ABOVE decision was not delayed by the word below and LEFT was not delayed by right. A second experiment, in which subjects matched word meaning against word location, showed that "different" responses were delayed whenever the representations of the word and location shared vertical or horizontal features. No asymmetries were found in this experiment. Seymour (1973) concluded that both location and word meaning are represented as sets of components or features rather than in a unitary form. If locations were represented in an undifferentiated manner as an abstract symbol or name, then one would expect that all incongruent words would interfere equally, and that all "different" decisions would be equally difficult. The finding that there is greater difficulty when the incongruent, irrelevant information is on the same dimension as the relevant suggests that dimensions are important aspects of the coding of visual locations as well as words.

Both of these experimental paradigms which have been used to find evidence for the notion that linguistic and perceptual codes share common components suffer from the fact that they encourage the linguistic processing of nonlinguistic events. The Clark et al. (1973) experiments require comparisons of pictures with words, while Stroop-like tasks usually require a linguistic response to nonlinguistic stimuli (a spoken word or the selection of a verbally labeled key). However, nonlinguistic information may be represented in an analogue form under a different set of circumstances. The question that is of interest here concerns auditory space and is twofold: (1) When subjects are encouraged to encode auditory space linguistically, are the codes composed of discrete dimensions, as are aspects of visual space, or are they unitary? (2) When subjects are not encouraged to encode auditory space linguistically, is there any evidence of a nonlinguistic code? Two experiments investigate these questions by using an auditory spatial Stroop-like task, in which subjects hear the words front, back, left, or right delivered by audio speakers located at the FRONT, BACK, LEFT, or RIGHT of the subject. The location of the word is relevant and requires a four-choice response, while the irrelevant word meaning may be congruent with location, incongruent but on the same dimension, incongruent and on a different dimension, or neutral with respect to location. If auditory space is coded in terms of dimensions, then one would expect more interference from incongruent words defined by the same dimension as the location than those defined by a different dimension.

Table 1 presents a possible system for defining the locative terms used here. It is derived mainly from Leech (1969). All of the terms share the (-VER) feature, which refers to the horizontal axis (as opposed to the vertical axis, which is primary). The front/back dimension is considered the primary horizontal axis (+PRI), while the left/right dimension is secondary (-PRI). Front is unmarked $(\rightarrow$ PLUS), back is marked ( $\leftarrow$ PLUS). Olson and Laxar (1973) have provided evidence that right is

Table 1

Possible Sy stem for Defining Locative Terms and their Corresponding Locations

\begin{tabular}{ll} 
Front & - VER + PRI $\rightarrow$ PLUS \\
Back & -VER + PRI $\leftarrow$ PLUS \\
Left & - VER - PRI $\rightarrow$ PLUS \\
Right & -VER - PRI $\leftarrow$ PLUS \\
\hline
\end{tabular}


unmarked ( $\rightarrow$ PLUS) while left is marked ( $\leftarrow$ PLUS). Since the horizontal dimension is shared by all of the terms, one would expect each incongruent word to interfere with a decision about its location. But since the specific horizontal dimension is different for front/back and left/right, one would expect more interference between front and back and between left and right than from one horizontal dimension to the other. Furthermore, since front and right are unmarked and back and left are marked, one might also expect more interference when the ( $\rightarrow$ PLUS) or ( $\leftarrow$ PLUS) feature is shared. Pairs of terms sharing two features (i.e., front/back, left/right, front/right, and back/left) should produce the most interference. Pairs sharing only the (-VER) feature should produce less interference. These predictions are derived from the hypothesis that auditory space is encoded in terms of the discrete dimensions used to define locative terms and presumably visual space. If, on the other hand, the code for auditory space is unitary, one would expect the interference effects of all of the incongruent words to be equal.

Two experiments were conducted. The first encouraged linguistic processing by requiring that decisions about location be made by selecting a verbally labeled key. The second experiment attempted to eliminate linguistic encoding by having unlabeled keys spatially arranged to correspond with the locations of the stimulus events. Previous studies (see, e.g., Palef, 1978; Pritchatt, 1968) have demonstrated a reduction or elimination of interference from words to decisions about nonlinguistic events when the decisions can be made nonlinguistically. This is presumably because no linguistic stage of processing is required, and words will interfere only with linguistic codes. A complete elimination of facilitation and interference effects in Experiment 2 would support the claim that the auditory code used was nonlinguistic.

\section{EXPERIMENT 1}

\section{Method}

Subjects. Twenty-four University of Toronto undergraduates served as subjects. Each was paid $\$ 2.50 / \mathrm{h}$.

Apparatus. The stimuli were presented by means of a Teac four-channel recorder and four speakers located directly in FRONT and BACK of the subject and to his LEFT and RIGHT. Each prerecorded auditory word started a Gerbrands Klockounter, which was stopped when the subject pressed one of four labeled response keys arranged in a row in front of him. A panel of lights, each illuminated when a given response key was pressed, permitted the experimenter to check the accuracy of responses.

Procedure and Design. Each trial consisted of the presentation of three warning clicks, followed after a 1 -sec delay by one of the words, front, back, left, and right, and a 4-sec intertrial interval. The warning occurred on all four speakers; the test word occurred on only one speaker, in the location FRONT, BACK, LEFT, or RIGHT. In the location condition, the subjects were required to decide the location of the word while attempting to ignore its meaning, and to indicate their responses by pressing the appropriate key as rapidly and accurately as possible. On congruent trials, the meaning of the word was the same as its location, e.g., left presented at the LEFT. On incongruent trials, the meaning and location differed, e.g., left on the RIGHT. Neutral trials consisted of the presentation of the word $X$ on one speaker. A word meaning condition was also included in which subjects responded to the meaning of the word and attempted to disregard its location. [Sometimes, spatial locations interfere with decisions about words. The direction of interference seems to depend on the relative processing speeds of the two information sources (Palef \& Olson, 1975)]. Congruent and incongruent trials were defined as above. Neutral trials consisted of the presentation of a locative term on all four speakers simultaneously.

A different group of 12 subjects each performed the word meaning and location parts of the experiment. Each subject was tested in one session consisting of 240 trials, of which the first 10 were considered practice and were not included in the analysis. An equal number of congruent, incongruent, and neutral trials were presented in random order. Each of the four relevant locations or location words also occurred randomly with equal probability. Assignment of locations and location words to response keys was counterbalanced across subjects.

\section{Results}

The reaction time results for the location and word meaning conditions are presented in Figure 1. The error rates were approximately $5 \%$ and $2 \%$ in the two conditions, respectively. The low error rates justified eliminating error trials from the analysis.

Figure 1 shows both facilitation and interference effects in the location condition, but no such effects in the word meaning condition. Analysis of variance performed on the location data showed a significant effect of congruency $[F(2,16)=46.96, p<.001]$. Neuman-Keuls tests on individual means showed significant differences $(p<.01)$ between the congruent and neutral conditions and between the incongruent and neutral conditions, and this was true for each of

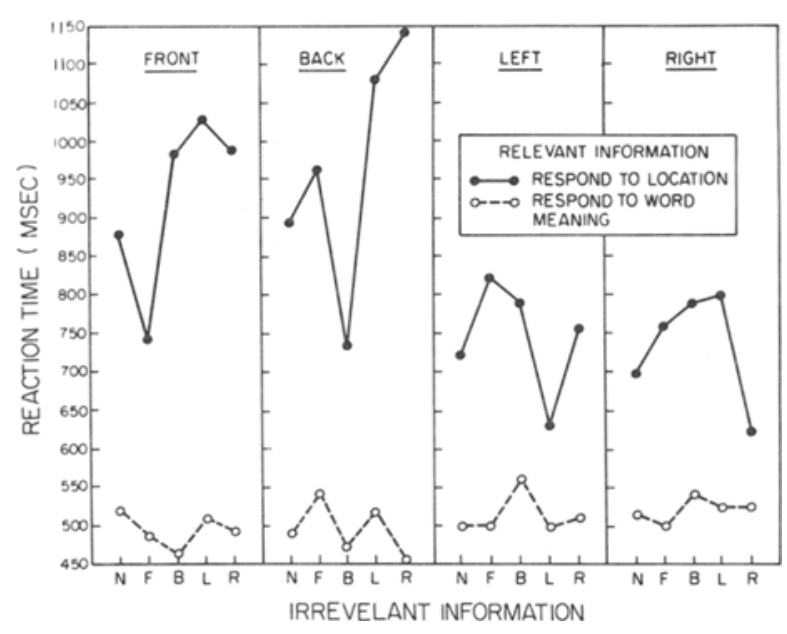

Figure 1. Mean reaction time for responses to location and to word meaning classified by the response made and the nature of the irrelevant information (Experiment 1). $N=$ neutral, $F=$ front, $B=$ back, $L=$ left, $\mathbf{R}=$ right. 
the four locations. There was also a significant effect for locations $[F(3,24)=7.01, p<.01]$. NeumanKeuls tests $(p<.05)$ showed that reaction times to LEFT and RIGHT were faster than to FRONT and BACK, but there were no significant differences between LEFT and RIGHT or between FRONT and BACK. Neutral reaction times to locations LEFT and RIGHT were approximately $180 \mathrm{msec}$ faster than reaction times to FRONT and BACK. Finally, there was a significant Congruency by Location interaction $[F(6,48)=2.99, p<.05]$, since the amount of interference and facilitation was greater for the locations FRONT and BACK than for LEFT and RIGHT. As for the word-meaning condition, analysis of variance showed no significant effect of congruency $[F(2,16)=1.14$, n.s. $]$ and no effect for word meaning $[\mathrm{F}(3,24)=.61$, n.s. $]$.

Let us look more specifically at the amount of interference provided by incongruent words on judgments of location. Analyses of variance were performed on the incongruent data for each location separately. Three out of four showed no significant differences in the effects of different incongruent words on locations. There was a significant effect of word meaning on responses to the location BACK $[\mathrm{F}(2,22)=5.74, \mathrm{p}<.01]$, with right providing more interference than front $[\mathrm{t}(11)=2.86, \mathrm{p}<.05]$. The word right shares only one feature with BACK, i.e., (-VERT), whereas front shares two features with BACK, i.e., ( - VERT) and (+PRI). Therefore, this difference is contrary to the prediction presented above that greater overlap of features would produce greater interference.

\section{EXPERIMENT 2}

The purpose of Experiment 2 was to eliminate the need for linguistic coding of locations by making the spatial arrangement of the response keys compatible with the spatial locations of the stimuli. One might then expect an elimination of facilitation and interference effects from irrelevant words. This result, together with the findings of Experiment 1, would support the conclusion that auditory location information can be represented in a format that is different from that for words under one set of circumstances, and in a linguistic-like format under a different set of circumstances.

The method was basically the same as in Experiment 1 , except that the response keys were unlabeled and were spatially arranged to correspond with the four possible locations of the word. They were arranged at the FRONT, BACK, LEFT, or RIGHT of a center point, at which the subject rested his index finger in preparation for responding. The left and right keys were clearly more compatible with the LEFT and RIGHT locations of the stimulus than were the front and back keys with the FRONT and BACK locations. Twelve subjects participated in this

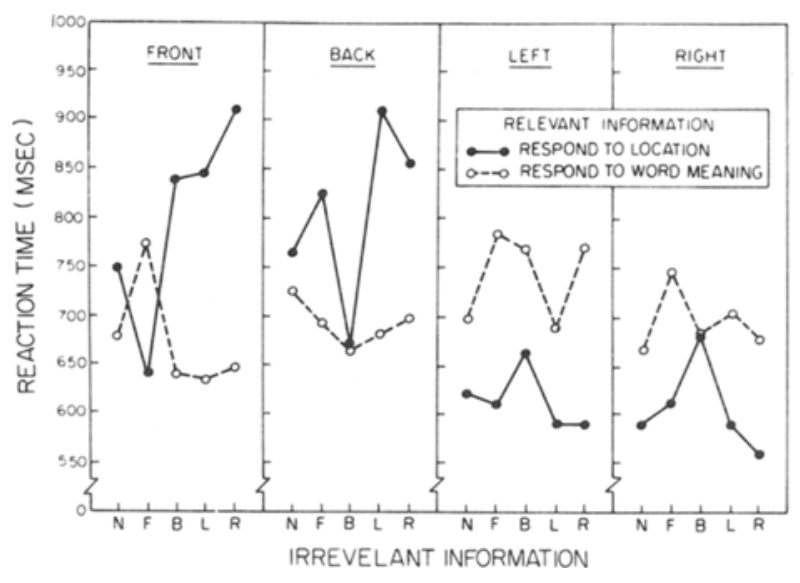

Figure 2. Mean reaction time for responses to location and to word meaning classified by the response made and the nature of the irrelevant information (Experiment 2). $\mathbf{N}=$ neutral, $\mathbf{F}=$ front, $\mathbf{B}=$ back, $\mathbf{L}=$ left, $\mathbf{R}=$ right.

experiment, half responding to the locations of the word and half to word meaning. All other aspects of the procedure and design were the same as in Experiment 1.

\section{Results}

The reaction time results are presented in Figure 2 for both responses to word meaning and word location. Error rates were less than 5\%. It is clear that there are facilitation and interference effects in the location condition but not in the word meaning condition. An analysis of variance on the location data showed a significant effect of congruency $[F(2,10)=$ $14.07, \mathrm{p}<.01$ ], a significant effect of location $[F(3,15)=7.26, p<.01]$, and also a significant Congruency by Location interaction $[F(6,30)=5.14$, $p<.01$ ], suggesting that the effect of congruency was greater for the positions FRONT and BACK than for LEFT and RIGHT. In fact, Neuman-Keuls tests done on individual means showed no significant $(p<.05)$ interference or facilitation effects for locations LEFT and RIGHT, but did show significant facilitation and interference effects for FRONT and BACK. Neuman-Keuls tests $(p<.05)$ also showed that reaction times to locations FRONT and BACK were not significantly different, that reaction times to locations LEFT and RIGHT were not significantly different, but that all other comparisons were different. As for the word-meaning data, an analysis of variance showed no effect of congruency, but did show a significant effect for word meaning $[F(3,15)$ $=9.71, p<.01]$. Neuman-Keuls tests $(p<.05)$ showed that reaction time for responding to the word front was significantly slower than to the words right, back, or left.

One may investigate the amount of interference provided by the various incongruent words on judgments of location only for locations FRONT and 
BACK, since only in these conditions are the effects of congruency significant. Analyses of variance were performed on the incongruent data for these two locations separately, neither of which showed any significant differences among the effects of the different words. There was no evidence that the number of overlapping dimensions in the definitions of the word and location determined the amount of interference between them. This replicated the findings of Experiment 1.

\section{Discussion}

Two experiments demonstrated that when subjects judge the auditory location of a word whose meaning is semantically incongruent with its location, the amount of interference provided by the word is unrelated to the degree of semantic overlap between the word and location. In studies of visual space (Seymour, 1973), it has been shown that interference is maximal when locative terms and visual locations share common semantic components. Furthermore, marked locations which are linguistically more complex than unmarked ones take longer to encode from visual displays (Clark et al., 1973), presumably because they require additional representational components. No evidence was found for such differences in the auditory presentations of Experiments 1 and 2. Together, these results point to the conclusion that when visual space is encoded linguistically, the code is composed of discrete dimensions, whereas the code for auditory space appears to be unitary. It is important to point out that Leech's (1969) system for defining locative terms is only one possible model. Although it appears to describe the linguistic encoding of visual space, other dimensions might be important for auditory space. It is not clear what these dimensions might be, however. In any case, it remains a possibility that auditory space is encoded along as yet unspecified dimensions.

Some other findings in Experiments 1 and 2 require explanation. First of all, in Experiment 1, there were no facilitation or interference effects from incongruent locations on judgments of word meaning. This is evidence of the fact that one of the necessary conditions for Stroop-like effects is that the irrelevant information is processed faster or at least as fast as the relevant information (Palef \& Olson, 1975). Since, in Experiment 1, neutral reaction time to the words was faster than to locations, word meaning interfered with and facilitated decisions about location, but locations did not affect word meaning decisions. In Experiment 2, words were processed faster than locations FRONT and BACK, but more slowly than locations LEFT and RIGHT. Therefore, words interfered with locations FRONT and BACK, but not with LEFT and RIGHT. Furthermore, locations interfered with the words left and right, although this effect did not reach significance.
Experiment 2 attempted to eliminate the need for linguistic processing of locations by using unlabeled response keys arranged spatially to correspond to the possible locations of the stimulus. This manipulation reduced neutral reaction time by approximately $140 \mathrm{msec}$ for locations FRONT and BACK and by approximately $100 \mathrm{msec}$ for LEFT and RIGHT compared to the times in Experiment 1. It also increased word reaction time by approximately $200 \mathrm{msec}$, presumably because it decreased the stimulus-response compatibility for word-meaning decisions. The interesting findings are the reduction in the magnitude of the facilitation and interference effects of words on locations FRONT and BACK (approximately $50 \mathrm{msec}$ for each effect), and the elimination of word effects on locations LEFT and RIGHT. The latter effect is confounded by the fact that words are processed more slowly than locations LEFT and RIGHT; hence, they cannot affect them. However, the reduction in the magnitude of the effects for locations FRONT and BACK when the amount of linguistic processing necessary for responding is presumably decreased suggests that aspects of auditory space may be coded in a manner that is unlike linguistic encoding. The evidence would have been more convincing if the manipulation had eliminated the facilitation and interference effects entirely. As suggested above, the front/back response keys were not highly compatible with the FRONT/BACK locations of the stimuli, since the former were arranged around a center point in front of the subject, while the latter were defined with respect to the subject himself. Hence, FRONT and BACK probably required some linguistic processing. More highly compatible stimulus-response connections might have resulted in a total elimination of the effects of irrelevant words.

In summary, the present investigation points to the conclusion that when auditory space is encoded linguistically, the code is a unitary, undifferentiated one as opposed to the set of features or dimensions that characterizes the code for visual space. Furthermore, it is suggested that auditory space may also be encoded in a nonlinguistic fashion, just as aspects of visual space are thought to be encoded sometimes in terms of analogue representations (Kosslyn \& Pomerantz, 1977). How other aspects of auditory events, such as time, are encoded is a matter for future investigation.

\section{REFERENCES}

Clark, H. H. Space, time, semantics, and the child. In T. E. Moore (Ed.), Cognitive development and the acquisition of language. New York: Academic Press, 1973.

Clark, H. H., Carpenter, P. A., \& Just, M. A. On the meeting of semantics and perception. In W. G. Chase (Ed.), Visual information processing. New York: Academic Press, 1973.

Greenberg, J. H. Language universals. The Hague: Mouton. 1966. 
Kosslyn, S. M., \& Pomerantz, J. R. Imagery, propositions and the form of internal representations. Cognitive Psychology, $1977,9,52-76$.

LeEch, G. Towards a semantic description of English. London: Longmans Green, 1969.

McLuhan, M. Understanding media:. The extensions of man. New York: McGraw-Hill, 1964.

Olson, D. R., \& Bialystock, E. Explorations in inner space: Aspects of the nature and development of spatial cognition. Hillsdale, N.J: Erlbaum, in press.

Olson, G. M., \& LAXAR, K. Asymmetries in processing the terms "right" and "left." Joumal of Experimental Psychology, 1973, 100, 284-290.

Palef, S. R. Judging pictorial and linguistic aspects of space. Memory \& Cognition, 1978, 6, 70-75.

PAleF, S. R., \& Olson, D. R. Spatial and verbal rivalry in a
Stroop-like task. Canadian Journal of Psychology, 1975, 29, 201-209.

PritchatT, D. An investigation into some of the underlying associative verbal processes of the Stroop colour effect. Quarterly Journal of Experimental Psychology, 1968, 20, 351-359.

SEYMOUR, P. H. K. Stroop interference in naming and verifying spatial locations, Perception \& Psychophysics, 1973, 14, 95-100. Stroop, J. R. Studies of interference in serial verbal reactions. Journal of Experimental Psychology, 1935, 18, 643-661.

Williams, D. C. Acoustic space. In E. S. Carpenter (Ed.), Explorations: Studies in culture and communication. Toronto: University of Toronto Press, 1955.

(Received for publications July 11, 1977; revision accepted February 27, 1978.) 